\title{
Modified preparation, and characteristic of vermiculite as catalysts support for catalytic pyrolysis
}

\author{
Yiqian Wang ${ }^{1, \mathrm{a}}$, Zhipeng Sun ${ }^{1, \mathrm{~b}^{*}}$ \\ ${ }^{1}$ Materials and Energy school, Guangdong University of Technology, Guangzhou, Guangdong, 511400, China
}

\begin{abstract}
Natural vermiculite, used as layered catalyst support, was modified with different chemical methods, such as acid treatment, introducing into $\mathrm{Na}^{+}$and organic modification. The vermiculite-based catalysts were characterized by SEM, BET and XRD respect to their images, textures and structures. SEM results presented big pieced of lamellate vermiculite which had complete structure and piled tightly, and the expansion of layer of modified vermiculites. According to XRD results, it had been found that a characteristic peak appeared at $2^{\circ}$ compared with natural vermiculite, and the spacing of layers of the modified samples increased obviously. These results corresponded with BET results which modified vermiculites had larger surface area and pore diameter than natural sample. It meant that modification was beneficial to broaden layered structure and modified vermiculites had the potential as catalyst support.
\end{abstract}

\section{Introduction}

China possesses various vermiculite resource, especially Xinjiang Yili county is the largest vermiculite deposit, and its reservoir is ranked the second in the world. Vermiculite is a water-containing aluminosilicate mineral, and its structural is made up of two siliconoxygen tetrahedrons and an octahedron sandwiched ${ }^{[1]}$. The interlayer structure consists of water molecules and exchangeable cations. Some water molecules form coordination octahedrons around the interlayer cations, occupying specific positions in the structure, while some water molecules are in free state ${ }^{[2]}$. The interlayer cations are mainly $\mathrm{Mg}^{2+}$ and $\mathrm{Ca}^{2+}$, other cations such as $\mathrm{Na}^{+}, \mathrm{K}^{+}, \mathrm{Cs}^{+}, \mathrm{Li}^{+}, \mathrm{Rb}^{+}$may exist. The cations in the octahedral sheet are mainly $\mathrm{Mg}^{2+}, \mathrm{Al}^{3+}, \mathrm{Fe}^{3+}, \mathrm{Ni}^{+}$. Due to water molecule and exchangeable cations exist in the expandable interlayer of vermiculite, it has excellent adsorption capacity and ion exchange capacity, which can exchange the cation with $\mathrm{H}^{+}$in the acid solution, and then change the distribution of acid amount. At the same time, it also has great heat expansibility, stable chemical properties and special layers characteristics of the structure, thus can be applied in environmental protection, such as preparing adsorbent for the waste gas or contaminated wastewater and catalyst carrier. At present, expanded vermiculite are widely used to optimize the physical and chemical properties of vermiculite to apply in various fields. Domestic and foreign scholars have conducted a series of modifications for vermiculite ${ }^{[3]}$ to change its structure and improve performance. Structure characteristics and the interlayer region of vermiculite have provided a good place for chemical reaction. Specifically, when foreign ions or compounds are introduced into the interdomain, that will change the spacing of layers, and the charge of tetrahedron and octahedron, also destroy the charge balance of the vermiculite layer, ultimately change that structure and properties ${ }^{[4]}$. Currently, vermiculite is usually modified by thermal expansion, interlamination insertion and other methods. Studies have shown that after modification the physical properties such as the adsorption capacity and expansibility have greatly improved, and the scope of application of vermiculite is expanded ${ }^{[5]}$. Liu et. have reported that after acid treatment the most dissolved amount cations are $\mathrm{K}, \mathrm{Ca}$, $\mathrm{Na}$, secondary are $\mathrm{Mg}, \mathrm{Fe}, \mathrm{Al}$. To balance the surface charge, Lewis active sites will be formed in the surface. There will be many pits existed in the surface and interlayer of vermiculite with the increasing of the surface area. Besides, the ion exchange capacity of vermiculite corresponds with the interlayer ion species ${ }^{[6]}$. The higher cation electricity price and hydration energy the vermiculite has, the stronger the adsorption capacity it gets, and the most difficult to be exchanged. Thus, sodium modification can improve its ion exchange properties and exchange capacity, which can broaden the application pathways of vermiculite ${ }^{[7]}$. The organic modification utilizes the exchangeable characteristics of water molecules and cations to introduce organic cations into the interlayer to expand the distance of the layer and change the hydrophobicity of the inner and outer surfaces of vermiculite clay, forming organic intercalation vermiculite. Owing to these perfect physical and chemical characteristics and their cheap, environment friendly and rich in resources features, it is an ideal catalyst support. Therefore, it is desirable to generate high surface area catalyst by modifying the chemical character of the support.

b* Zhipeng Sun: zpsunxj@gdut.edu.cn 


\section{Experimental}

\subsection{Material}

Natural vermiculite (200 mesh) purchased in SihmaAldrich (Shanghai) company was used as supported material of the catalyst. The hydrochloric acid $(36.5 \% \mathrm{wt})$ was purchased from Guangzhou Chemical Reagent Factory, and sodium chloride (AR, 99.5\%) was purchased from Aladdin reagent Shanghai Co., Ltd. Besides, hexadecyltrimethylammonium bromide was purchased from Tianjin Guangfu Fine Chemical Research Institute. All reagents were purchased as analytical grade chemicals, which were used as modifier in the experiments and all experimental solutions were prepared by distilled water.

\subsection{Catalyst preparation}

After acidic treatment, $\mathrm{Al}^{3+}$ cations would be leached from the tetrahedron sheets of vermiculite and stabilization potential of interlayer cations could be reduced. Firstly, 3g natural vermiculite mixed with 0.6 $\mathrm{M}$ nitric acid stirring at magnetic stirrer for $5 \mathrm{~h}$ at $75^{\circ} \mathrm{C}$. Subsequently, the clay was separated from the solution and washed with demineralised water until $\mathrm{pH}$ 7. Then, the acidic vermiculite (Acid-Verm) was obtained under $650^{\circ} \mathrm{C}$ high temperature calcination. Cations, adsorbed in the vermiculite layer and balanced the substitution ion of aluminum in the tetrahedral sheet, could be replaced by external cations and when exchanged with $\mathrm{Na}^{+}$, the cation exchange capacity of vermiculite greatly increased. In the next step, the modified clay could be converted into the sodium form by ion exchange. 100 $\mathrm{mL}$ saturated sodium solution added $3 \mathrm{~g}$ vermiculite, stirring at bath heating reaction for $6 \mathrm{~h}$ under $80^{\circ} \mathrm{C}$ water. Then the Na vermiculite (Na-Verm) was separated from the solution, dried at room temperature, calcined and finally obtained. Organic intercalation/pillaring meant that organic molecules/ions entered the interlayer of clay through ion exchange or directly entered the interlayer of clay and changed the microenvironment. Therefore, the organic vermiculite (Organic-Verm) was obtained by the following steps. A certain amount of vermiculite added distilled water to form $2 \mathrm{wt} \%$ suspension, heating at $80^{\circ} \mathrm{C}$ and stirring well until evenly dispersed. After adding 1.5 CEC hexadecyltrimethylammonium bromide at the suspension, stirred at $80^{\circ} \mathrm{C}$ for $2 \mathrm{~h}$, centrifuged, washed 3 times with deionized water, dried in $50^{\circ} \mathrm{C}$ oven, and passed through a 200-mesh sieve for later use.

\subsection{Catalysts characterization}

In this study, we used an acoustic spectrometer Hitach SU8200 to observe the surface structure. Micrometrics ASAP 2010 nitrogen gas adsorption analyzer was used to determine the textural parameters. Before the analysis, the samples were degassed under vacuum at $300^{\circ} \mathrm{C}$ for $8 \mathrm{~h}$. The surface area was calculated by the BET, and micropore volume of the samples was determined by the $\mathrm{t}$-method of DeBoer. XRD patterns were collected on a
RigakuUltima IV diffractometer using $\mathrm{Cu} / \mathrm{Ka}$ radiation source at $40 \mathrm{kV}$ and $20 \mathrm{~mA}$ with a scan rate of 0.5 degree/min to survey the crystal structure of materials.

\subsection{Catalytic test and analysis}

These vermiculite-based catalysts respectively mixed with plastic samples at a ratio of $10 \%$ (mass fraction) and together put into the pyrolysis reactor (TL-1200 tube furnace purchased by Nanjing Boyuntong Instrument Co., Ltd.). The weight of each plastic sample was about $10 \mathrm{~g}$ each time, which mixed polyethylene and polypropylene. Firstly, the system was heated to $300^{\circ} \mathrm{C}$ at a rate of $30^{\circ} \mathrm{C} / \mathrm{min}$ and kept it for 10 minutes. Then, the temperature was increased to $480^{\circ} \mathrm{C}$ at a rate of $10^{\circ} \mathrm{C} / \mathrm{min}$ and kept it for 20 minutes. And then the generated gas vapor were purged to gas bag and condensable oil product was dissolved by acetone. The yield of solid residue (excluding catalyst) and gas products were calculated by GC.

\section{Results}

\subsection{SEM analysis}

The micro surface morphology and structure of the vermiculite and Organic-Verm were observed using SEM. It was aimed to be micro analysis of the change of surface structure in vermiculite after organic modification. From Fig.1a, natural vermiculite had a complete sheet-like structure, large particle size and a small specific surface area. The layered structure remained better after organic modification, which was a complete layered structure. Due to the characteristic vermiculite had, the organic solution entered the layer and adsorbed in inner wall layer during the processing, which is not easily rinsed in the washing process.

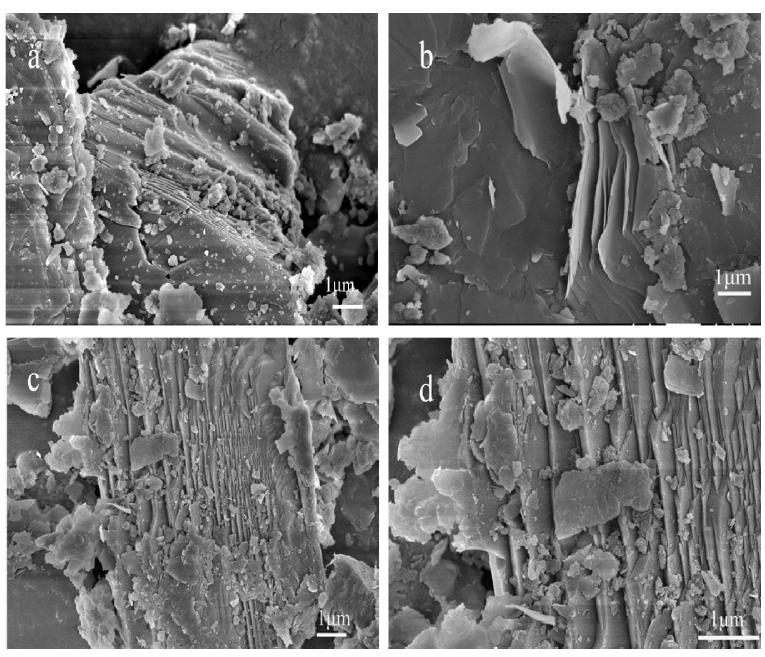

Fig.1 SEM image of natural vermiculite $(a, b)$ and OrganicVerm (c, d).

\subsection{XRD analysis}

X-ray diffractograms (XRD) of the modification process of vermiculite were measured to study their crystallinity. 
According to Fig. 2, we could see that there was an obvious diffraction peaks at $9.1^{\circ}$, which was the diffraction peak d002. After acid treatment, the degree of crystallization increased with the increase of acid concentration from $0.1 \mathrm{~mol} / \mathrm{L}$ to $0.6 \mathrm{~mol} / \mathrm{L}$ while the shape of peak was widened and the structure was destroyed at $0.8 \mathrm{~mol} / \mathrm{L}$ HCL. Meanwhile, the basal spacing decreased from $1.014 \AA$ to $1.0 \AA$, suggesting that appropriate hydrochloric acid could remove the impurity in vermiculite while the high acidity destroyed the structure of material. Fig. 3 exhibited the XRD results of sodium and organic modification samples. From the picture, we could see that the characteristic peak appeared at $2^{\circ}$ after modification, which might be the symbol of the intercalation. Besides, the d002 peak shifted to low degree and the spacing changed to $1.0 \AA$ and $0.98 \AA$, lower than the original vermiculite $(1.014 \AA)$. It meant that the deposition of surfactants and cosurfactants in the clays interlayer resulted in the $\mathrm{d} 002$ peak shifting to lower angles. This effect was related to the increase of interlayer distance in the vermiculite. It was mainly attributed to $\mathrm{Na}^{+}$successfully insert the interlayer of vermiculite and change the crystalline phase. These results were in line with the BET surface area mentioned as follow. In addition, the introduction of organic modifier into the layered clay led to significantly different change. The d002 peak became sharp, distinct and well-defined, indicating a good crystallinity for this sample. According to XRD patterns, we revealed that acid treatment, introducing into $\mathrm{Na}^{+}$and organic modification both could increase the interlayer distance of vermiculite and organic modification was the best.

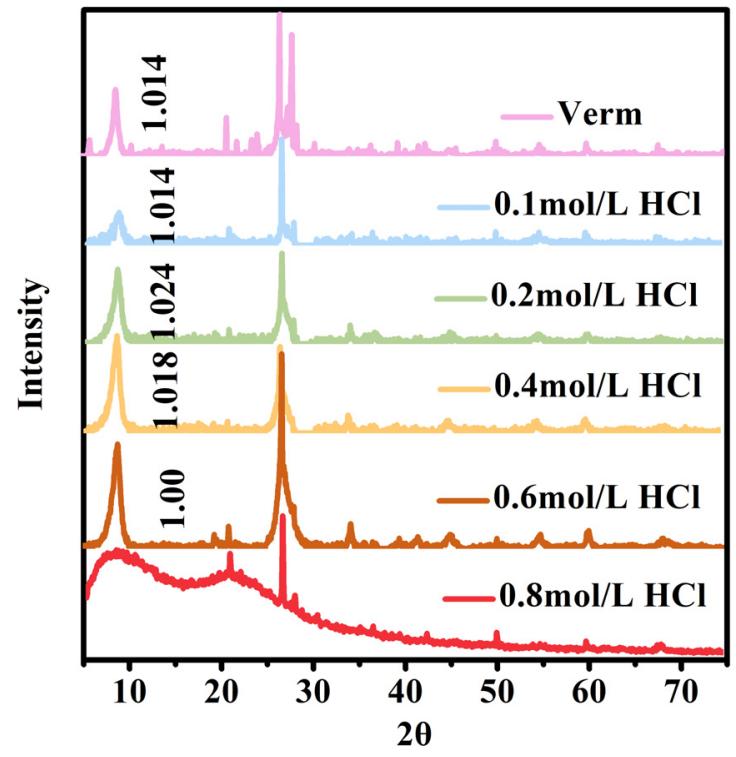

Fig.2 XRD pattern of vermiculite after different acid concertation.

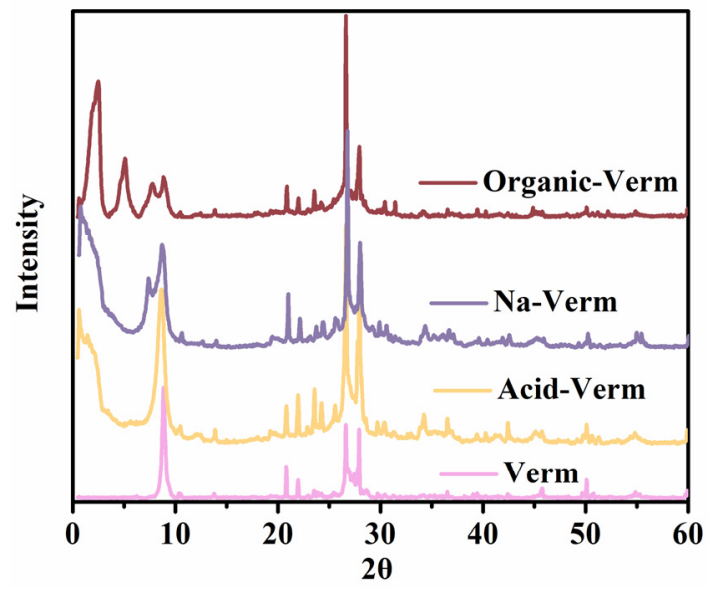

Fig.3 XRD pattern of vermiculite after different modifications.

\subsection{BET analysis}

Table 1 showed textural parameters of the samples. Natural vermiculite showed BET and pore diameter of $5.63 \mathrm{~m}^{2} \cdot \mathrm{g}^{-1}$ and $18.8 \mathrm{~nm}$ respectively, which meant that it was a low porosity material and most of mesopores located in the external surface of natural vermiculite. But the surface area of Acid-Verm was $42.1 \mathrm{~m}^{2} \cdot \mathrm{g}^{-1}$, revealing that dilute hydrochloric acid could exchange with cations. It's said that due to the radius of $\mathrm{H}^{+}$was significantly smaller than the $\mathrm{Ca}^{2+}$ and $\mathrm{Mg}^{2+}$, the pore diameter exited in the vermiculite shrunk, and smaller pore size resulted in higher specific surface area. As a result of the sodium modification improved its ion exchangeable properties, $\mathrm{Na}$-Verm showed surface area and pore size were 33.7 $\mathrm{m}^{2} \cdot \mathrm{g}^{-1}$ and $7.0 \mathrm{~nm}$, respectively. Meanwhile, the BET surface area of Organic-Verm was highest, comparing with the other samples, which has expanded the layer distance and could expose more active sites for improving the activity of catalyst. The BET surface areas of all the modification catalysts were larger than that of natural material, which also evidenced that the significant role of modification methods.

Table 1. Textural properties of vermiculite after modification.

\begin{tabular}{|c|c|c|c|c|}
\hline & Verm & $\begin{array}{c}\text { Acid- } \\
\text { Verm }\end{array}$ & $\begin{array}{c}\text { Na- } \\
\text { Verm }\end{array}$ & $\begin{array}{c}\text { Organic } \\
\text {-Verm }\end{array}$ \\
\hline $\mathrm{S}\left(\mathrm{m}^{2} \cdot \mathrm{g}^{-1}\right)$ & 8.49 & 42.1 & 33.7 & 63.5 \\
\hline $\mathrm{V}\left(\mathrm{cm}^{3} \cdot \mathrm{g}^{-1}\right)$ & 0.04 & 0.065 & 0.06 & 0.083 \\
\hline $\begin{array}{c}\text { Pore diameter } \\
(\mathrm{nm})\end{array}$ & 18.8 & 6.2 & 7.0 & 52.4 \\
\hline
\end{tabular}

\subsection{Pyrolysis yields}

Table 2 showed the gas composition obtained from modified samples (detected by GC). The collected pyrolysis gas was consisted of $\mathrm{C}_{1}-\mathrm{C}_{5}$ mixed gaseous hydrocarbons. It could be observed that $\mathrm{C}_{3}$ was the main fraction of pyrolysis gases in catalytic pyrolysis. After different modifications, the selectivity of gas product changed slightly, which might be due to the surface area 
and pore structure changed. According to Table 3, the results showed that among the currently tested catalysts, modified vermiculite had higher heavy hydrocarbons

$\left(>\mathrm{C}_{13}\right)$ yields. This might be due to the acidity of these catalysts which was influenced by structure. Comparing with natural vermiculite, the composition of oil products cracked from modified vermiculite-based catalysts mainly concentrated on heavy hydrocarbons. Organic -Verm presented a particularly high selectivity for the heavy hydrocarbons fraction, which was higher than Acid-Verm and Na-Verm. These results further indicated the modified vermiculite had the good potential to be applied in producing valuable diesel fuel from plastic waste because of the hydrocarbons in the range of $>\mathrm{C}_{13}$ were representative of diesel products.

Table 2. Composition of the pyrolysis gas (vol\%).

\begin{tabular}{|c|c|c|c|c|c|}
\hline & $\mathrm{C}_{1}$ & $\mathrm{C}_{2}$ & $\mathrm{C}_{3}$ & $\mathrm{C}_{4}$ & $\mathrm{C}_{5}$ \\
\hline Verm & 3.1 & 17.0 & 38.6 & 5.6 & 35.6 \\
\hline $\begin{array}{c}\text { Acid- } \\
\text { Verm }\end{array}$ & 7.3 & 14.6 & 50.8 & 1.2 & 25.7 \\
\hline $\begin{array}{c}\text { Sodium- } \\
\text { Verm }\end{array}$ & 12.4 & 22.2 & 35.0 & 17.4 & 12.5 \\
\hline $\begin{array}{c}\text { Organic- } \\
\text { Verm }\end{array}$ & 11.3 & 20.9 & 33 & 15.8 & 10.9 \\
\hline
\end{tabular}

Table 3. Main components of the oil product determined by $\mathrm{GC}(\%$ area).

\begin{tabular}{|c|c|c|}
\hline & $\begin{array}{c}\text { Light } \\
\text { hydrocarbons } \\
\left(\leq \mathrm{C}_{13}\right)\end{array}$ & $\begin{array}{c}\text { heavy } \\
\text { hydrocarbons } \\
\left(>\mathrm{C}_{13}\right)\end{array}$ \\
\hline Verm & 53.2 & 46.8 \\
\hline Acid-Verm & 42.3 & 57.7 \\
\hline Sodium-Verm & 30.7 & 69.3 \\
\hline Organic-Verm & 23.4 & 76.6 \\
\hline
\end{tabular}

\section{Conclusions}

The modified samples were obtained from acid treated, $\mathrm{Na}^{+}$introduced and organic intercalated. The surface image, textural as well as structural parameters of these samples were determined and compared with the parameters of natural vermiculite. The modified samples were proved to be the high selectivity catalysts for diesel oil. The best results were obtained from Organic-Ver attributed to the expansion of the interlayer spacing. In summary, we have researched that the change of structure and catalytic performance in catalyst support, and provided the possibility for further exploration of the industrial application of modified catalysts. Because the stable and large surface area of catalyst support provided large interlayer spacing and exposed a great deal of active sites.

\section{Acknowledgments}

We acknowledged the financial support from the Guangdong Province of science and technology program 2018A050506079.

\section{References}

1. Chmielarz, L., Kuśtrowski, P., Michalik, M., Dudek, B., Piwowarska, Z., Dziembaj. R. (2008) Vermiculites intercalated with $\mathrm{Al} 2 \mathrm{O} 3$ pillars and modified with transition metals as catalysts of DeNOx process. Catal. Today, 137: 242-246.

2. Ahenach, J., Cool, P., Vansant, E.F. (2000) Enhanced Brnsted acidity created upon Al-grafting of porous clay heterostructures ia aluminium acetylacetonate adsorption. J. Phys. Chem. Chem. Phys., 2: 5750-5755.

3. Dorsan, S., Elizabeth, M.S., Cláudio, N.R., Gisele, T.R., Augusto, F.S. (2019). New sodium activated vermiculite process. Testing on $\mathrm{Cu} 2+$ removal from tailing dam waters. J Hazard Mater, 366: 34-38.

4. Antonakou, E., Lappas, A., Nilsen, M.H., Bouzga, A., Stocker, M. (2006) Evaluation of various types of Al-MCM-41 materials as catalysts in biomass pyrolysis for the production of bio-fuels and chemicals. Fuel, 85: 2202-2212.

5. Chmielarz, L., Kuśtrowski, R., Piwowarska, Z., Dudek, B., Gil, B., Michalik, M. (2009) Montmorillonite, vermiculite and saponite based porous clay heterostructures modified with transition metals as catalysts for the DeNOx process. J. Appl. Catal., B, 88: 331-340.

6. Aguado, J., Serrano, D.P., Escola, J.M., Peral. A. (2009) Catalytic cracking of polyethylene over zeolite mordenite with enhanced textural properties, J. Anal. Appl. Pyrol., 85: 352-358.

7. Kelkar, S., Saffron, C.M., Andreassi, K. (2015) A survey of catalysts for aromatics from fast pyrolysis of biomass. Appl. Catal. B-Environ., 174:85-95. 